\title{
Translating DPYD genotype into DPD phenotype: using the DPYD gene activity score
}

The dihydropyrimidine dehydrogenase enzyme (DPD, encoded by the gene DPYD) plays a key role in the metabolism of fluoropyrimidines. DPD deficiency occurs in 4-5\% of the population and is associated with severe fluoropyrimidine-related toxicity. Several SNPs in DPYD have been described that lead to absent or reduced enzyme activity, including $D P Y D * 2 A, D P Y D * 13, c .2846 \mathrm{~A}>\mathrm{T}$ and c.1236G $>\mathrm{A} /$ haplotype B3. Since these SNPs differ in their effect on DPD enzyme activity, a differentiated dose adaption is recommended. We propose the gene activity score for translating DPYD genotype into phenotype, accounting for differences in functionality of SNPs. This method can be used to standardize individualized fluoropyrimidine dose adjustments, resulting in optimal safety and effectiveness.

Keywords: capecitabine $\bullet$ dihydropyrimidine dehydrogenase $\bullet$ DPYD • 5-fluorouracil - fluoropyrimidines $\bullet$ gene activity score $\bullet$ individualized medicine $\bullet$ pharmacogenomics

The fluoropyrimidine anticancer drug 5-fluorouracil (5-FU) and its oral prodrug capecitabine are frequently used in the treatment of a variety of cancers, including breast, colorectal, head and neck and gastric cancer. The dihydropyrimidine dehydrogenase enzyme (DPD), encoded by the gene DPYD, plays a key role in the metabolism of fluoropyrimidines. Over $80 \%$ of the administered dose of 5-FU is metabolized by DPD in the liver into the inactive metabolite 5,6-dihydro5-fluorouracil, which makes DPD the rate-controlling enzyme for inactivation of 5 -FU [1]. DPD deficiency occurs in 4-5\% of the population and results in decreased inactivation of 5-FU. This can lead to an increase in active metabolites of 5-FU which is associated with an increased risk of severe and even fatal toxicity [2-4]. Toxicity could be limited by exposing DPD-deficient patients to a decreased dose of fluoropyrimidines, to keep plasma levels of 5-FU and its metabolites at a therapeutic level for these patients. Over 30 genetic polymorphisms in DPYD have been described among which several lead to reduced function or a nonfunctional
DPD enzyme [4-6]. Polymorphisms can appear in heterozygous form (one SNP on one allele), homozygous form (two identical SNPs on two alleles) or double heterozygous form (two different SNPs on either one or two alleles, the latter is also called compound heterozygous). Two SNPs on two alleles lead to a larger decrease in DPD enzyme activity, compared with the heterozygous form. An example of a $D P Y D$ polymorphism is the splice-site variant $D P Y D^{*} 2 A$ (IVS14+1G>A; c.1905+1G>A; rs3918290), which leads to deletion of exon 14 and hence a nonfunctional DPD enzyme and is the most studied polymorphism in $D P Y D$.

In recent years, genotyping costs have dropped significantly and pre-emptive testing for single or multiple SNPs to guide treatment with fluoropyrimidines has become accessible. Upfront genotype-directed dose adaptation of fluoropyrimidines is feasible and has been shown to increase safety for patients and to be cost-effective for $D P Y D^{*} 2 A[7,8]$. However, only a minority of institutions have implemented screening programs as standard of care [9-11]. Some physicians are reluctant
Linda M Henricks ${ }^{\dagger, 1,2}$, Carin ATC Lunenburg ${ }^{\dagger, 3}$, Didier Meulendijks ${ }^{1,2}$, Hans Gelderblom ${ }^{3}$, Annemieke Cats ${ }^{4}$, Jesse J Swen ${ }^{5}$, Jan HM Schellens ${ }^{1,2,6}$ \& Henk-Jan Guchelaar*,5

'Division of Clinical Pharmacology, Department of Medical Oncology, The Netherlands Cancer Institute, Amsterdam, The Netherlands ${ }^{2}$ Department of Molecular Pathology, The Netherlands Cancer Institute, Amsterdam, The Netherlands ${ }^{3}$ Department of Medical Oncology, Leiden University Medical Center, Leiden, The Netherlands

${ }^{4}$ Department of Gastroenterology \& Hepatology, The Netherlands Cancer Institute, Amsterdam, The Netherlands ${ }^{5}$ Department of Clinical Pharmacy \& Toxicology, Leiden University Medical Center, P.O. Box 9600, 2300 RC Leiden, The Netherlands

${ }^{6}$ Division of Pharmacoepidemiology \& Clinical Pharmacology, Department of Pharmaceutical Sciences, Faculty of Science, Utrecht University, Utrecht, The Netherlands

*Author for correspondence:

Tel.: +31715262790

Fax: +31715266980

h.j.guchelaar@lumc.nl

${ }^{\dagger}$ Authors contributed equally 
to implement upfront genotype-guided dosing due to a lack of results from prospective randomized studies comparing genotype-guided and traditional dosing. The only prospective randomized study was terminated prematurely for ethical reasons as one patient in the control arm died due to 5-FU-related toxicity [12].

In addition to $D P Y D^{*} 2 A$, other SNPs in $D P Y D$ have been described to result in decreased DPD enzyme activity, including $D P Y D^{*} 13$ (c.1679T>G; I560S; rs55886062), c.2846A>T (D949V; rs67376798) and c.1236G>A (E412E; rs56038477, in haplotype B3) [1315]. However, not all of these SNPs result in a similar decrease in DPD enzyme activity as $D P Y D^{*} 2 A[3,14,16]$. As a result of the growing number of alleles and their range of activity, deriving DPD phenotype from genotype is increasingly challenging. In the near future the number of alleles will increase even further, since genetic testing is developing fast and single SNP testing might be replaced by testing SNP panels, whole exome sequencing or even whole genome sequencing. Consequently, there is a need for an individualized recommendation of dose adjustment of fluoropyrimidines, taking into account the specific genetic variants and their resulting reductions in DPD enzyme activity. In this paper, we describe a method for translation of DPYD genotype into DPD phenotype making use of the gene activity score. This method accounts for the differences in functionality of the SNPs in DPYD, which results in a more differentiated dose adjustment and thus in optimal safety and effectiveness.

\section{Previous guidelines \& recommendations}

According to the US FDA and EMA capecitabine and 5-FU are contraindicated in patients with a known DPD deficiency $[17,18]$. However, no recommendations are given for upfront screening for DPD deficiency and no distinction is made between heterozygous or homozygous DPD-deficient patients. Also the American Society of Clinical Oncology, European Society for Medical Oncology and National Comprehensive Cancer Network do not state any genotyping guidelines or recommendations prior to fluoropyrimidine treatment. In the guideline of the Clinical Pharmacogenetics Implementation Consortium (a network that provides guidelines on the translation of genetic laboratory tests into actionable prescribing decisions) patients heterozygous for $D P Y D^{*} 2 A, D P Y D^{*} 13$ or c. $2846 \mathrm{~A}>\mathrm{T}$ are considered to have intermediate or partial DPD enzyme activity and recommended for these patients is an initial dose reduction of at least $50 \%$ (no dosing recommendations are given for other SNPs, including c.1236G $>\mathrm{A}$, because evidence on these variants was considered weak or conflicting) [19]. Also the Pharmacogenetics Working Group of the Royal Dutch Society for the Advancement of Pharmacy (KNMP) has provided guidelines. They recently updated their online guidelines for dose adjustments for fluoropyrimidines from a $50 \%$ dose reduction for heterozygous carriers to more specified dose reductions of 25 or $50 \%$ in heterozygous carriers of a SNP in DPYD (depending on the specific SNP), and 50,75 or $100 \%$ in patients carrying more than one SNP in DPYD [20,21]. We consider the dosing guidance of the Clinical Pharmacogenetics Implementation Consortium and KNMP very useful and would like to add the gene activity score to these guidelines. With the gene activity score we can facilitate in a more specific dose adjustment in fluoropyrimidine treatment using current knowledge on differences in DPD enzyme activity due to DPYD variants.

\section{Known DPYD alleles \& their effect on DPD enzyme activity $D P Y D * 2 A(r s 3918290)$}

$D P Y D^{*} 2 A$ is the most widely studied polymorphism in DPYD. The SNP was first described by Vreken et al. in a case series of two unrelated patients [22] and McLeod et al. named it $D P Y D^{*} 2 A$ in an article in which the nomenclature for a series of DPYD SNPs was defined [23]. Allele frequencies of $D P Y D^{*} 2 A$ have been reported to vary between $\sim 0.1$ and $1.0 \%$ in African-American and Caucasian populations, respectively $[13,19,24,25]$. DPYD $2 A$ leads to skipping of the entire exon 14 and deletion of 165 base pairs which results in a truncated protein that is catalytically inactive $[22,26]$. This was recently confirmed in a study by Offer $e t a l$. where in an in vitro model of DPD activity several DPYD variants were homozygously expressed in mammalian cells and the enzymatic activity of expressed protein was completely absent [27]. This indicates that in heterozygous carriers of this variant, who have one dysfunctional allele and one functional allele, $\sim 50 \%$ of the normal DPD enzyme activity will remain. Furthermore, a correlation between the $D P Y D^{*} 2 A$ variant and reduced enzyme activity in peripheral blood mononuclear cells (PBMCs) was found in several ex vivo studies that confirmed decreased function of $D P Y D^{*} 2 A$ [26,28-30] and consequently an association was also found between $D P Y D^{*} 2 A$ and reduction in fluoropyrimidine clearance in patients [31,32]. In numerous studies an association between $D P Y D^{*} 2 A$ allele carriership and the increased risk of toxicity related to fluoropyrimidine treatment was confirmed [4,24,31,33-45]. For example, in a meta-analysis by Terrazzino et al. a strong correlation between the $D P Y D^{*} 2 A$ allele and overall grade $\geq 3$ toxicity was found (odds ratio 5.42, p < 0.001) [33]. Deenen et al. described a mean capecitabine dose 
reduction of $50 \%$, guided by toxicity, in patients carrying $D P Y D^{*} 2 A$, compared with a mean dose reduction of $10 \%$ in wild-type patients [42]. Also, an initial dose reduction of capecitabine or $5-\mathrm{FU}$ of $50 \%$ of standard dose has proven to decrease the risk of severe toxicity in $D P Y D^{*} 2 A$ carriers $[7,8]$. The above-mentioned in vitro, ex vivo and in vivo studies provide solid evidence for the nonfunctionality of $D P Y D^{*} 2 A$ and a $50 \%$ reduced function in patients heterozygous for $D P Y D^{*} 2 A$.

\section{c. 2846A $>\mathrm{T}(\mathrm{rs} 67376798)$}

The c.2846A $>\mathrm{T}$ variant allele was first described by van Kuilenburg et al. in 2000 [28]. The c.2846A>T polymorphism leads to a structural change in the DPD enzyme that interferes with cofactor binding or electron transport [16]. Reported allele frequencies of c. $2846 \mathrm{~A}>\mathrm{T}$ vary from 0.1 to $1.1 \%$ in African-Americans and Caucasians, respectively $[13,19,24,46]$. In vitro data show that homozygous expression of the c.2846A $>\mathrm{T}$ variant results in an activity of 59\% compared with wildtype $(\mathrm{p}=0.0031)$ [13]. Although the enzyme activity of c. $2846 \mathrm{~A}>\mathrm{T}$ is significantly impaired, it is not comparable to the extent observed for $D P Y D^{*} 2 A$, where homozygous expression resulted in a completely nonfunctional enzyme [27]. This finding that homozygous expression of c. $2846 \mathrm{~A}>\mathrm{T}$ results in $\sim 50 \%$ reduction, suggests that a heterozygous carrier would have around $25 \%$ reduction in DPD activity. Furthermore, also in clinical practice a difference between the effect of the $D P Y D^{*} 2 A$ variant and the c. $2846 \mathrm{~A}>\mathrm{T}$ variant has been observed. Deenen et al. described an average $25 \%$ dose reduction for c.2846A>T heterozygous patients in response to fluoropyrimidine-related toxicity, compared with $50 \%$ for $D P Y D^{*} 2 A$ heterozygous patients [42]. Although there are less publications for c. $2846 A>T$ than for $D P Y D^{*} 2 A$, several studies and two meta-analyses found an association between the c. $2846 \mathrm{~A}>\mathrm{T}$ variant and increased risk of severe fluoropyrimidine-associated toxicity, which indicates that a dose reduction is warranted $[4,24,33,36,41,42,44,45,47]$. In the study by Rosmarin et al. an odds ratio of 9.35 $(p=0.0043)$ was found between c.2846A $>T$ and capecitabine-related severe ( $\geq$ grade 3 ) toxicity [47]. The evidence described above shows that c.2846A $>\mathrm{T}$ has rest-activity left, but that a dose reduction would still be required to prevent toxicities that would occur using a full dose of fluoropyrimidines. Therefore, based upon the available evidence we can assume that a dose reduction of $25 \%$ is most rational.

\section{$D P Y D * 13$ (rs55886062)}

$D P Y D^{*} 13$ was first described by Collie-Duguid et al. as 'T1679G' [48]. The allele frequency was found to vary from 0.07 to $0.1 \%$ in Caucasians [19,24]. The precise functional consequences of the $D P Y D^{*} 13$ variant have not yet been unraveled, but are thought to be related to destabilization of a sensitive region of the protein [16]. $D P Y D^{*} 13$ has been found in patients with decreased enzyme activity, not in patients showing normal DPD enzyme activity [29]. Homozygous expression of this variant resulted in a 75\% reduction of DPD enzyme activity compared with wild-type, as reported in an in vitro study by Offer $e t$ al. [27]. This suggests that this variant almost completely inactivates the protein. Decreased DPD enzyme activity in patients with the $D P Y D^{*} 13$ variant was determined only in a limited number of ex vivo studies using PBMCs $[16,29,30,48]$. A major variation of enzyme activity was found, ranging from 1.7 times to 500 times decreased as compared with the normal enzyme activity and once the enzyme activity was undetectable [30], although it must be mentioned that these results could be influenced by other copresent $D P Y D$ variants. Patients with $D P Y D^{*} 13$ showed severe toxic side effects in several studies $[4,24,29,44,48,49]$. Also, dose adjustments were described by two groups [4,24]. Morel et al. described a heterozygous patient that experienced severe grade 4 toxicity. After a 6-week treatment interruption, 5-FU was safely reintroduced with individual pharmacokinetic adjustment, based on 5-FU plasma levels [4]. The above-mentioned studies show that $D P Y D^{*} 13$ results in an almost nonfunctional enzyme and consequently low enzyme activity levels. Without a dose reduction, toxicities are likely to develop, however safe use of 5-FU is still possible with a dose adjustment. We suggest a starting dose of $50 \%$ for patients carrying $D P Y D^{*} 13$ to ensure safe and effective use of fluoropyrimidines.

\section{c.1236G >A/HapB3 (rs56038477)}

The c.1236G $>$ A variant was first described by Seck et al. as a silent mutation that displays normal DPD enzyme activity [46]. The c.1236G $>$ A polymorphism occurs in exon 11 and is a synonymous variant that is in complete linkage with c. $483+18 \mathrm{G}>\mathrm{A}$, c. $680+139 \mathrm{G}>\mathrm{A}$, c. $959-51 \mathrm{~T}>\mathrm{G}$ and c.1129-5923C $>\mathrm{G}$ [14]; these variants in linkage have been termed haplotype B3 [14,15]. The c.1129-5923C>G intronic polymorphism (rs75017182) results in aberrant splicing and is likely to be the responsible variant for the effect on DPD enzyme activity [3,14]. The frequency of heterozygous patients in Caucasian populations was reported to vary between 2.6 and $6.3 \%[14,15,42,49,50]$. DPD enzyme activity for c.1236G>A carriers was measured in PBMCs in two studies $[14,46]$. Enzyme activities were reported to be $2.9,4.2,6.2$ and $1.6 \mathrm{nmol} /\left(\mathrm{mg}^{*} \mathrm{~h}\right.$ ) (normal value $\left.=9.6 \pm 2.6 \mathrm{nmol} /\left[\mathrm{mg}^{*} \mathrm{~h}\right]\right)$ for one homozygous and three heterozygous carriers of c.1236G>A, respectively [14]. In addition, a heterozygous patient in 
another study was found to have an enzyme activity of $10.2 \mathrm{nmol} /\left(\mathrm{mg}^{*} \mathrm{~h}\right)$, which was reported as 'normal activity,' since the enzyme activity of the population ranged from 4.8 to $15 \mathrm{nmol} /\left(\mathrm{mg}^{*} \mathrm{~h}\right)$ [46]. Unfortunately data on c.1236G>A and enzyme activity are limited and not consistent. The homozygous patient still had $30 \%$ DPD activity remaining [17]. Furthermore we observed two homozygous patients with this variant in our own institute with a relevant DPD enzyme activity left of around $50 \%$, showing that this variant does not result in a completely nonfunctional enzyme (Author's Unpublished Data). In the study of Sistonen et al. the ratio between endogenous dihydrouracil (DHU) and uracil (U) was measured in patients carrying the c.1129-5923C>G variant [50]. This ratio can be used as a phenotyping marker for DPD enzyme activity, as described in several studies [51-55]. Sistonen et al. found a statistically significant decrease in DHU/U ratio compared with wild-type patients $(\mathrm{p}=0.044)$. However, no significant effect for the other $D P Y D$ risk variants $\left(D P Y D^{*} 2 A, D P Y D^{*} 13\right.$ and c. $\left.2846 \mathrm{~A}>\mathrm{T}\right)$ was observed, which might be caused by the small sample size of patients with those variants. The c.1236G $>$ A/HapB3 variant has been associated with severe and lethal toxicity $[14,15,42,49,56]$. For example, Froehlich et al. found a relative risk of $3.74\left(\mathrm{p}=2 \times 10^{-5}\right)$ in c. $1236 \mathrm{G}>\mathrm{A} / \mathrm{HapB} 3$ carriers for severe toxicity (grade 3-5) [49]. In contrast, no significant effect of the $.1236 \mathrm{G}>\mathrm{A} / \mathrm{HapB} 3$ variant was found in two other studies $[44,47]$. A dose reduction to prevent toxicity may be advantageous since multiple studies found a correlation with severe toxicity; however the degree of dose reduction cannot easily be determined with the enzyme activity from only two published studies and conflicting results in clinical studies. In heterozygous patients, a dose reduction of $50 \%$ would be too large since c. $1236 \mathrm{G}>\mathrm{A} / \mathrm{HapB} 3$ does not result in a completely nonfunctional enzyme. No dose reduction at all would be in contradiction to the correlation found between this variant and toxicity. Therefore a more cautious dose reduction of 25\% seems appropriate, to avoid both increased risk of toxicity and prevent underdosing.

Also our own experimental data support the differentiation between various SNPs in DPYD. We determined the endogenous pretreatment ratio between DHU and $U$ in a large cohort of patients $(n=539)$ treated with capecitabine or 5-FU (manuscript in preparation). This cohort is a subset of patients participating in a prospective multicenter trial of $D P Y D^{*} 2 A$ guided dosing of fluoropyrimidines (clinicaltrials.gov identifier: NCT00838370) [7,8]. The DHU and U levels were measured in pretreatment serum samples using a validated LC-MS/MS method (manuscript in preparation), chromatographic separation was performed on an Acquity UPLC ${ }^{\circledast}$ HSS T3 column $(150 \times 2.1 \mathrm{~mm}$ ID, particle size $1.8 \mu \mathrm{m}$ ) and a triple quadruple mass spectrometer (API5500, AB Sciex, USA) was used for quantification of $U$ and DHU. The method was validated over a concentration range of $1-100 \mathrm{ng} / \mathrm{ml}$ for $\mathrm{U}$ and $10-1000 \mathrm{ng} / \mathrm{ml}$ for DHU. Genotyping for DPYD variants was performed using standard PCR methods. A distinction was made between patients heterozygous for $D P Y D^{*} 2 A$, c. $2846 \mathrm{~A}>\mathrm{T}, D P Y D^{*} 13$ or c. $1236 \mathrm{G}>\mathrm{A}$ and wild-type patients (Figure 1). For patients heterozygous for $D P Y D^{*} 2 A, \quad$ c. $2846 \mathrm{~A}>\mathrm{T}, \quad D P Y D^{*} 13$ and c.1236G $>\mathrm{A}$ the median relative $\mathrm{DHU} / \mathrm{U}$ ratio compared with wild-type is $52,68,50$ and $101 \%$, respectively. These results confirm that DPD enzyme activity differs between carriers of certain DPYD polymorphisms and points toward a differentiated dose reduction for each individual SNP.

\section{Gene activity score}

The gene activity score method is based on the principle that variant alleles can differ in the extent to which they influence enzyme activity. Such a method was first described by Steimer et al. where a 'quantitative functional gene dose' is assigned to alleles of the gene CYP2D6, a highly polymorphic gene that is involved in the metabolism of various clinically used drugs, including antidepressants, antipsychotics and opioids [57]. Thereafter Gaedigk et al. introduced the 'activity score' and divided CYP2D6 alleles in three categories, consisting of fully functional alleles (value of 1), reduced activity alleles (value of 0.5 ) and nonfunctional alleles (value of 0 ) [58]. The values for both alleles of a patient are summed, leading to an individual gene activity score that represents the enzymatic phenotype of the patient. This method results in a uniform way of describing phenotypes and can be used for adjusting the dose of a drug. For CYP2D 6 it has been demonstrated that the gene activity score is valid and easy-to-use for translating genotype and predicted phenotype [58]. The gene activity score may also be useful to properly interpret different DPD enzyme activities, translate these into a phenotype and thus personalize fluoropyrimidine treatment according to DPYD genotype. With this tool a more precise distinction between nonactive and reduced activity alleles can be made and it also provides the possibility to include novel SNPs which may be identified in the near future using whole exome and whole genome sequencing. The activity score as proposed by Gaedigk et al. has proven beneficial for CYP2D6, for which a large number of polymorphisms are known.

We have fully investigated and described four SNPs in DPYD $\left(D P Y D^{*} 2 A\right.$, c. $2846 \mathrm{~A}>\mathrm{T}, D P Y D^{*} 13$, c.1236G $>$ A/HapB3). This literature review describes 


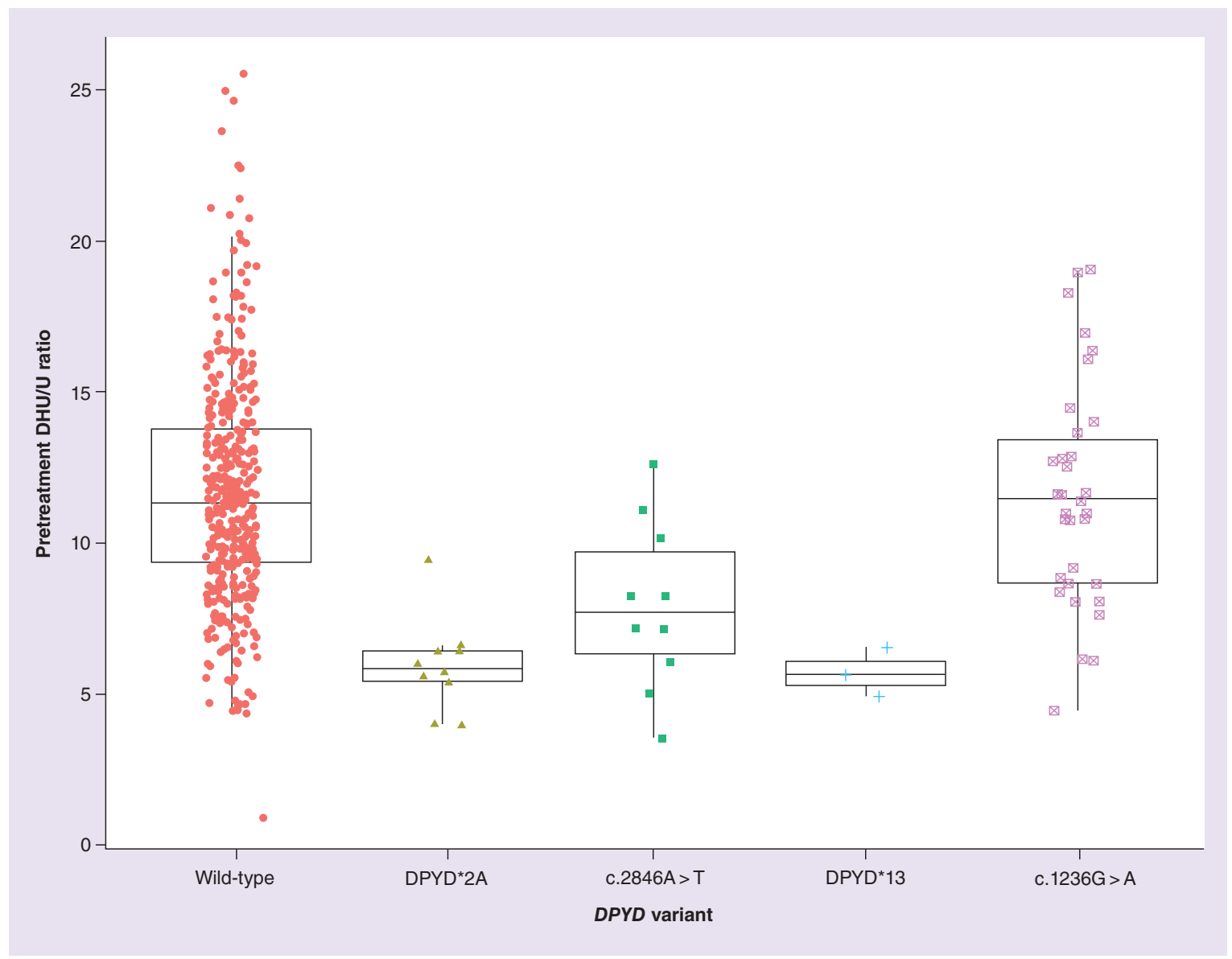

Figure 1. DHU/U ratio according to DPYD genotype. Shown are individual values and a box plot with the median of the DHU/U ratio for patients with a DPYD polymorphism or DPYD wild-type patients.

DHU: Dihydrouracil; U: Uracil.

what DPD enzyme activities are to be expected in patients with a certain SNP in DPYD. In addition to that, we have shown additional data of pretreatment $\mathrm{DHU} / \mathrm{U}$ ratio in correlation to $D P Y D^{*} 2 A$, c. $2846 \mathrm{~A}>\mathrm{T}$, $D P Y D^{*} 13$ and c.1236G $>$ A. We focus on these four SNPs because, based on the available literature data, we believe they are the most relevant. Additional SNPs can be easily added to the gene activity score in the future when sufficient data are available. An outline for the suggested assigned values to various alleles of $D P Y D$ is given in Table 1. So far only the four SNPs described above are included, because sufficient evidence is available that they result in low DPD enzyme activity and severe fluoropyrimidine-related toxicity. Consequently, following the calculated gene activity scores for DPYD an individualized dose recommendation for fluoropyrimidines can be given, as is shown in Table 2. This is a recommendation for a starting dose; after the first or second cycle the dose can be titrated according to tolerance. Wild-type patients have two fully functional alleles, are allocated the maximal gene activity score of 2 and will receive the standard starting dose. Patients heterozygous for $D P Y D^{*} 2 A$ or $D P Y D^{*} 13$ have one nonfunctional allele and one fully functional allele, will therefore have an expected DPD enzyme activity of $50 \%$ and receive a gene activity score of 1. The recommended dose reduction of capecitabine or 5 -FU for those patients is $50 \%$. Patients carrying one allele with the c.2846A $>\mathrm{T}$ or c. $1236 \mathrm{G}>\mathrm{A} / \mathrm{HapB} 3$ variant will have one decreased activity allele and one fully functional allele, which results in DPD enzyme activity of $\sim 75 \%$ of normal. They are allocated a gene activity score of 1.5 , for which a recommended starting dose of $75 \%$ of the standard dose applies.

\section{Discussion \& conclusion}

There is ample evidence that shows that DPD-deficient patients develop severe toxicities when treated with a normal dose of fluoropyrimidines. Even though this relation is widely known, there is no global systematic approach to prevent severe toxic side effects using $D P Y D$ polymorphisms as predictive markers. Upfront $D P Y D^{*} 2 A$ screening has been implemented in a limited number of institutions and other SNPs are 


\begin{tabular}{|c|c|c|}
\hline Activity value & Alleles & Ref. \\
\hline \multirow[t]{2}{*}{0} & $\begin{array}{l}D P Y D * 2 A \\
(\mathrm{rs} 3918290)\end{array}$ & {$[4,8,10,11,19,27,29-46]$} \\
\hline & $\begin{array}{l}D P Y D * 13 \\
(\text { rs55886062) }\end{array}$ & {$[4,19,30,32,33,46,49,50]$} \\
\hline \multirow[t]{2}{*}{0.5} & $\begin{array}{l}c .2846 A>T \\
(r s 67376798)\end{array}$ & {$[4,13,24,33,36,41,42,44,47]$} \\
\hline & $\begin{array}{l}\text { c.1236G >A/ } \\
\text { HapB3 } \\
\text { (rs56038477) }\end{array}$ & {$[14,15,42,44,46,47,49,50,56]$} \\
\hline 1 & $\begin{array}{l}D P Y D * 1 \\
\text { (wild-type) }\end{array}$ & \\
\hline
\end{tabular}

increasingly added to the standard genetic screening. Testing for an increasing number of SNPs that result in different DPD enzyme activities makes it harder to derive a dosing advice. The gene activity score is a new method for translating DPYD genotype into DPD phenotype. It can be used to standardize the process of describing DPD enzyme activity, which stimulates uniformity. In the CPIC guideline a dose recommendation of $50 \%$ is advised for $D P Y D^{*} 2 A, D P Y D^{*} 13$ and c. $2846 \mathrm{~A}>\mathrm{T}$ [19]. In the gene activity score as proposed in this manuscript we adopt these recommendations for $D P Y D^{*} 2 A$ and $D P Y D^{*} 13$, but deviate in the dose advice for c.2846A $>\mathrm{T}$ and include a dose advice for c.1236G $>\mathrm{A} / \mathrm{HapB} 3$. We have summarized in vitro, ex vivo and in vivo studies to determine the appropriate dose recommendation for these SNPs. In addition, we have shown our own experimental data. Our data are in agreement with previous data and show a $50 \%$ reduced DPD enzyme activity in patients heterozygous for $D P Y D^{*} 2 A$ and $D P Y D^{*} 13$ and an $25 \%$ decreased activity for heterozygous patients with c.2846A $>\mathrm{T}$. Unfortunately, our data on c.1236G >A do not correspond and additional data containing DPD enzyme activity measurements in patients with c.1236G>A/ $\mathrm{HapB} 3$ are scarce and not in agreement. Including our

\begin{tabular}{|c|c|}
\hline Gene activity score & $\%$ of standard dose \\
\hline 0 & Alternative drug \\
\hline 0.5 & 25 \\
\hline 1 & 50 \\
\hline 1.5 & 75 \\
\hline 2 & 100 \\
\hline
\end{tabular}

study, three out of four studies suggest that c.1236G >A results in an enzyme activity close to normal levels. However, Sistonen et al. showed a significant reduction in $\mathrm{DHU} / \mathrm{U}$ ratio in patients carrying this variant [50] and associations with the development of severe toxic side effects have also been described. The toxicity data point out that a dose reduction for c.1236G >A/HapB3 is required, but a dose reduction of $50 \%$ would be too large considering the measured enzyme activities. Therefore a dose reduction to $75 \%$ of the normal dose for heterozygous patients seems appropriate in order to prevent toxicity as well as to prevent underdosing. After the initial dose reduction the patient should be closely monitored and the dose can be adjusted according to occurring toxicity.

Currently only four SNPs in DPYD are allocated a gene activity score, since we consider these variants are the most relevant polymorphisms. It has been described before that 13 [59] to 19 [60] variants are expected to result in DPD deficiency. However, more research is necessary on the effect of these other SNPs on DPD enzyme activity before they can be included in the gene activity score. With the gene activity score approach it is possible to continuously keep adding variant alleles or updating the values of the gene activity score that are assigned to variant alleles. When new information on effects on enzyme activity is published, this can be included, while the currently proposed gene activity score can already be used in clinical practice. In addition, more research is needed with regard to compound heterozygous patients (patients who carry two different SNPs) and homozygous patients. These patients would benefit from an additional phenotyping test to measure the DPD enzyme activity as to determine the optimal dose adjustment or decide to treat with an alternative drug.

Both genotyping and phenotypic biomarkers have been proposed in order to predict and reduce toxicity in patients. However, the gold standard of phenotyping (measuring DPD enzyme activity in PBMCs) is not easy to implement as a routine test and other phenotyping methods, such as uracil test dose, endogenous $\mathrm{DHU} / \mathrm{U}$ ratio and $2-{ }^{13} \mathrm{C}$-uracil breath test, have not yet been fully validated or standardized [61]. Compared with phenotyping methods, genotyping methods are faster, easier and less expensive, so it is expected that it will be implemented more often as standard of care for patients undergoing fluoropyrimidine treatment.

The dose recommendations described in this article will be implemented in an upcoming large prospective clinical trial (NCT02324452) in the Netherlands where upfront genotypic assessment of DPYD will be performed for around 1250 patients treated with capecitabine or 5-FU. Simultaneously, our work was 
recently implemented by the Dutch Pharmacogenetics Working Group by using the gene activity score for translating DPYD genotype into DPD phenotype [21].

To conclude, we propose using the gene activity score for the translation of DPYD genotype into a numeric value that can be easily used to describe DPD phenotype and to advise an individualized dose adjustment for the use of fluoropyrimidines.

\section{Future perspective}

We expect that in the future more knowledge will be gained regarding relevant SNPs in DPYD other than the ones described in this article. Currently there are 13-19 SNPs expected to result in DPD deficiency. In addition, SNPs in other genes involved in fluoropyrimidine metabolism or mRNA could influence the DPD enzyme activity and could thus in the future be added to the activity score. The design of the gene activity score makes it possible to add other DYPD SNPs while main- taining a uniform method for describing DPD activity using a score table and for deriving individualized dose adjustments.

\section{Financial \& competing interests disclosure}

LM Henricks was supported by a grant from the Dutch Cancer Society and CATC Lunenburg was supported by an unrestricted grant from Roche Pharmaceuticals. There was no involvement in the study design, data collection, analysis or interpretation of the data. The members of the Pharmacogenetics Working Group of the Royal Dutch Society for the Advancement of Pharmacy (KNMP) are kindly acknowledged for scientific discussions. The authors have no other relevant affiliations or financial involvement with any organization or entity with a financial interest in or financial conflict with the subject matter or materials discussed in the manuscript apart from those disclosed.

No writing assistance was utilized in the production of this manuscript.

Executive summary

- Dihydropyrimidine dehydrogenase (DPD) plays a key role in the metabolism of fluoropyrimidines, a group of anticancer drugs including 5-fluorouracil and capecitabine.

- DPD-deficient patients are at increased risk for toxicity related to 5-fluorouracil or capecitabine.

- Genetic screening for polymorphisms in the DPYD gene resulting in DPD deficiency and genotypeguided adaptive dosing can prevent the risk of developing severe treatment-related toxicity.

Known DPYD alleles and their effect on DPD enzyme activity

- Based on literature review, we conclude that $D P Y D * 2 A$ and $D P Y D * 13$ result in a nonfunctional enzyme and thus in $50 \%$ residual activity of the DPD enzyme in heterozygotes.

- c.2846A $>$ T and c.1236G >A result in a partly dysfunctional enzyme and thus in $75 \%$ residual activity of the DPD enzyme in heterozygotes.

Gene activity score

- The gene activity score is a previously proposed method for the translation of CYP2D6 genotype to phenotype. We propose using the gene activity score for DPYD.

- With this method a distinction can be made between the various DPYD alleles and their functionality, resulting in a differentiated individualized dosing advice for fluoropyrimidines.

\section{Conclusion}

- Differentiation between individualized dosing of fluoropyrimidines is essential to reduce toxic side effects while maintaining efficacy.

- The gene activity score is a practical, easy to use tool that accounts for the differences in functionality of the SNPs in DPYD and accommodates a differentiated dose adjustment.

- The gene activity score can be implemented in its current form, while additional SNPs can easily be added in the future.

\section{References}

Papers of special note have been highlighted as:

- of interest; $\bullet \bullet$ of considerable interest

1 Longley D, Harkin D, Johnston P. 5-Fluorouracil: mechanisms of action and clinical strategies. Nat. Rev. Cancer 3(5), 330338 (2003).

2 Johnson MR, Diasio RB. Importance of dihydropyrimidine dehydrogenase (DPD) deficiency in patients exhibiting toxicity following treatment with 5-fluorouracil. Adv. Enzyme Regul. 41, 151-157 (2001).
3 Amstutz U, Froehlich TK, Largiadèr CR.

Dihydropyrimidine dehydrogenase gene as a major predictor of severe 5-fluorouracil toxicity. Pharmacogenomics 12(9), 1321-1336 (2011).

4 Morel A, Boisdron-Celle M, Fey L et al. Clinical relevance of different dihydropyrimidine dehydrogenase gene single nucleotide polymorphisms on 5-fluorouracil tolerance. Mol. Cancer Ther. 5(11), 2895-2904 (2006).

5 Del Re M, Di Paolo A, van Schaik RH et al. Dihydropyrimidine dehydrogenase polymorphisms 
and fluoropyrimidine toxicity: ready for routine clinical application within personalized medicine? EPMA J. 1(3), 495-502 (2010).

6 Hsiao HH, Lin SF. Pharmacogenetic syndrome of dihydropyrimidine dehydrogenase deficiency. Current Pharmacogenomics 5(1), 31-38 (2007).

7 Deenen MJ, Cats A, Mandigers CM et al. Prevention of severe toxicity from capecitabine, 5-fluorouracil and tegafur by screening for DPD-deficiency. Ned. Tijdschr. Geneeskd. 156(48), A4934 (2012).

8 Deenen MJ, Cats AJ, Sechterberger MK et al. Safety, pharmacokinetics (PK), and cost-effectiveness of upfront genotyping of DPYD in fluoropyrimidine therapy. J Clin Oncol. 29, Suppl Abstr. 3606 (2011).

- Interim analysis showing that individualized dosing based on upfront screening for $D P Y D^{*} 2 A$ is safe, feasible and cost-effective. Manuscript regarding this study is in preparation.

9 Boisdron-Celle M, Capitain O, Metges JP, Lecomte T, Matysiak-Budnik T, Morel A. Severe fluoropyrimidines toxicities: screen effectively for DPD deficiencies. Presented at: 8eme Congres de Physiologie, de Pharmacologie et de Therapeutique. Angers, France, 22-24 April 2013.

10 Siffert W. Pharmacogenetics in daily routine clinical practice. Presented at: 6th Santorini Conference Biologie Prospective 2012. Santorini, Greece, 30 September-2 October 2012.

11 Dunnenberger H, Crews K, Hoffman J et al. Preemptive clinical pharmacogenetics implementation: current programs in five US medical centers. Annu. Rev. Pharmacol. Toxicol. 55(1), 1-18 (2015).

12 B Boisdron-Celle M, Capitain O, Metges JP et al. Prevention of 5-FU-induced health-threatening toxicity by pretherapeutic DPD deficiency screening: medical and economic assessment of a multiparametric approach. Presented at: 2013 ASCO Annual Meeting. Chicago, USA, 31 May-14 June 2013.

13 Offer SM, Fossum CC, Wegner NJ, Stuflesser AJ, Butterfield GL, Diasio RB. Comparative functional analysis of DPYD variants of potential clinical relevance to dihydropyrimidine dehydrogenase activity. Cancer Res. 74(9), 2545-2554 (2014).

14 Van Kuilenburg AB, Meijer J, Mul AN et al. Intragenic deletions and a deep intronic mutation affecting pre-mRNA splicing in the dihydropyrimidine dehydrogenase gene as novel mechanisms causing 5-fluorouracil toxicity. Hum. Genet. 128(5), 529-538 (2010).

15 Amstutz U, Farese S, Aebi S, Largiadèr CR. Dihydropyrimidine dehydrogenase gene variation and severe 5-fluorouracil toxicity: a haplotype assessment. Pharmacogenomics 10(6), 931-944 (2009).

16 Van Kuilenburg AB, Dobritzsch D, Meinsma R et al. Novel disease-causing mutations in the dihydropyrimidine dehydrogenase gene interpreted by analysis of the threedimensional protein structure. Biochem. J. 364(Pt 1), 157-163 (2002).

17 US Food and Drug Administration. Pharmaocgenomic information capecitabine/fluorouracil (2015). www.fda.gov
18 European Medicines Agency. Summary of Product Characteristics, capecitabine (2015). www.ema.europa.eu

19 Caudle KE, Thorn CF, Klein TE et al. Clinical Pharmacogenetics Implementation Consortium guidelines for dihydropyrimidine dehydrogenase genotype and fluoropyrimidine dosing. Clin. Pharmacol. Ther. 94(6), 640-645 (2013).

-• Clinical Pharmacogenetics Implementation Consortium guideline: summarizing evidence regarding the associaton between $D P Y D$ variants and risk on toxicity and providing dosing recommendations for fluoropyrimidines based on genotype.

20 Swen JJ, Nijenhuis M, de Boer A et al. Pharmacogenetics: from bench to byte - an update of guidelines. Clin. Pharmacol. Ther. 89(5), 662-673 (2011).

21 Royal Dutch Society for the Advancement of Pharmacy. Background information Pharmacogenetics Dihydropyriminde dehydrogenase (DPD) (2015). www.kennisbank.knmp.nl

- Royal Dutch Society for the Advancement of Pharmacy guideline: Dutch guidelines on dose recommendations of fluoropyrimidines. They have recently updated their guidelines with the gene activity score.

22 Vreken P, Van Kuilenburg AB, Meinsma R et al. A point mutation in an invariant splice donor site leads to exon skipping in two unrelated Dutch patients with dihydropyrimidine dehydrogenase deficiency. J. Inherit. Metab. Dis. 19(5), 645-654 (1996).

23 McLeod HL, Collie-Duguid ES, Vreken P et al. Nomenclature for human DPYD alleles. Pharmacogenetics 8(6), 455-459 (1999).

24 Lee AM, Shi Q, Pavey E et al. DPYD variants as predictors of 5-fluorouracil toxicity in adjuvant colon cancer treatment (NCCTG N0147). J. Natl Cancer Inst. 106(12), pii: dju298 (2014).

25 Van Kuilenburg AB, Muller EW, Haasjes J et al. Lethal outcome of a patient with a complete dihydropyrimidine dehydrogenase (DPD) deficiency after administration of 5-fluorouracil: frequency of the common IVS14+1G $>A$ mutation causing DPD deficiency. Clin. Cancer Res. 7(5), 1149-1153 (2001).

26 Van Kuilenburg AB, Vreken P, Beex LV et al. Heterozygosity for a point mutation in an invariant splice donor site of dihydropyrimidine dehydrogenase and severe 5-fluorouracil related toxicity. Eur. J. Cancer. 33(13), 2258-2264 (1997)

27 Offer SM, Wegner NJ, Fossum C, Wang K, Diasio RB. Phenotypic profiling of DPYD variations relevant to 5-fluorouracil sensitivity using real-time cellular analysis and in vitro measurement of enzyme activity. Cancer Res. 73(6), 1958-1968 (2013).

- In vitro study investigating enzymatic activity of $D P Y D$ variants compared with wild-type.

28 Van Kuilenburg AB, Haasjes J, Richel DJ et al. Clinical implications of dihydropyrimidine dehydrogenase (DPD) deficiency in patients with severe 5-fluorouracil- 
associated toxicity: identification of new mutations in the DPD gene. Clin. Cancer Res. 6(12), 4705-4712 (2000).

in HH, Lee AM, Mattison LK, Diasio RB. Methylation of the DPYD promoter: an alternative mechanism for dihydropyrimidine dehydrogenase deficiency in cancer patients. Clin. Cancer Res. 11(24 Pt 1), 8699-8705 (2005).

30 Johnson MR, Wang K, Diasio RB. Profound dihydropyrimidine dehydrogenase deficiency resulting from a novel compound heterozygote genotype. Clin. Cancer Res. 8(3), 768-774 (2002).

31 Van Kuilenburg AB, Häusler P, Schalhorn A et al. Evaluation of 5-fluorouracil pharmacokinetics in cancer patients with a c.1905+1G >A mutation in DPYD by means of a Bayesian limited sampling strategy. Clin. Pharmacokinet. 51(3), 163-174 (2012).

32 Van Kuilenburg AB, Maring JG, Schalhorn A et al. Pharmacokinetics of 5-fluorouracil in patients heterozygous for the IVS14+1G > A mutation in the dihydropyrimidine dehydrogenase gene. Nucleosides Nucleotides Nucleic Acids 27(6), 692-698 (2008).

33 Terrazzino S, Cargnin S, Del Re M, Danesi R, Canonico PL, Genazzani AA. DPYD IVS14+1G>A and 2846A $>$ T genotyping for the prediction of severe fluoropyrimidinerelated toxicity: a meta-analysis. Pharmacogenomics 14(11), 1255-1272 (2013).

34 Magnani E, Farnetti E, Nicoli D et al. Fluoropyrimidine toxicity in patients with dihydropyrimidine dehydrogenase splice site variant: the need for further revision of dose and schedule. Intern. Emerg. Med. 8(5), 417-423 (2013).

35 Kristensen MH, Pedersen PL, Melsen GV, Ellehauge J, Mejer J. Variants in the dihydropyrimidine dehydrogenase, methylenetetrahydrofolate reductase and thymidylate synthase genes predict early toxicity of 5-fluorouracil in colorectal cancer patients. J. Int. Med. Res. 38(3), 870-883 (2010).

36 Schwab M, Zanger UM, Marx C et al. Role of genetic and nongenetic factors for fluorouracil treatment-related severe toxicity: a prospective clinical trial by the German 5-FU Toxicity Study Group. J. Clin. Oncol. 26(13), 2131-2138 (2008).

37 Salgado J, Zabalegui N, Gil C, Monreal I, Rodríguez J, García-Foncillas J. Polymorphisms in the thymidylate synthase and dihydropyrimidine dehydrogenase genes predict response and toxicity to capecitabine-raltitrexed in colorectal cancer. Oncol. Rep. 17(2), 325-328 (2007).

Largillier R, Etienne-Grimaldi M-CC, Formento J-LL et al. Pharmacogenetics of capecitabine in advanced breast cancer patients. Clin. Cancer Res. 12(18), 5496-5502 (2006).

39 Salgueiro N, Veiga I, Fragoso M et al. Mutations in exon 14 of dihydropyrimidine dehydrogenase and 5-Fluorouracil toxicity in Portuguese colorectal cancer patients. Genet. Med. 6(2), 102-107 (2004).

40 Van Kuilenburg AB, Meinsma R, Zoetekouw L, Van Gennip AH. High prevalence of the IVS14 + 1G>A mutation in the dihydropyrimidine dehydrogenase gene of patients with severe 5-fluorouracil-associated toxicity. Pharmacogenetics 12(7), 555-558 (2002)
41 Boisdron-Celle M, Remaud G, Traore S et al. 5-Fluorouracilrelated severe toxicity: a comparison of different methods for the pretherapeutic detection of dihydropyrimidine dehydrogenase deficiency. Cancer Lett. 249(2), 271-282 (2007).

42 Deenen MJ, Tol J, Burylo AM et al. Relationship between single nucleotide polymorphisms and haplotypes in DPYD and toxicity and efficacy of capecitabine in advanced colorectal cancer. Clin. Cancer Res. 17(10), 3455-3468 (2011).

43 Raida M, Schwabe W, Häusler P et al. Prevalence of a common point mutation in the dihydropyrimidine dehydrogenase (DPD) gene within the 5'-splice donor site of intron 14 in patients with severe 5 -fluorouracil (5-FU)related toxicity compared with controls. Clin. Cancer Res. 7(9), 2832-2839 (2001).

44 Loganayagam A, Arenas Hernandez M, Corrigan A et al. Pharmacogenetic variants in the DPYD, TYMS, CDA and MTHFR genes are clinically significant predictors of fluoropyrimidine toxicity. Br. J. Cancer. 108(12), 2505-2515 (2013).

45 Joerger M, Huitema AD, Boot $\mathrm{H}$ et al. Germline TYMS genotype is highly predictive in patients with metastatic gastrointestinal malignancies receiving capecitabine-based chemotherapy. Cancer Chemother. Pharmacol. 75(4), 763-772 (2015).

46 Seck K, Riemer S, Kates R et al. Analysis of the DPYD gene implicated in 5-fluorouracil catabolism in a cohort of Caucasian individuals. Clin. Cancer Res. 11(16), 5886-5892 (2005).

47 Rosmarin D, Palles C, Church D et al. Genetic markers of toxicity from capecitabine and other fluorouracil-based regimens: investigation in the QUASAR2 study, systematic review, and meta-analysis. J. Clin. Oncol. 32(10), 1031-1039 (2014).

- Trial and meta-analysis investigating polymorphisms in $D P Y D$ and $T Y M S$ and their association with toxicity.

48 Collie-Duguid ES, Etienne MC, Milano G, McLeod HL. Known variant DPYD alleles do not explain DPD deficiency in cancer patients. Pharmacogenetics 10(3), 217-223 (2000).

49 Froehlich TK, Amstutz U, Aebi S, Joerger M, Largiadèr CR. Clinical importance of risk variants in the dihydropyrimidine dehydrogenase gene for the prediction of early-onset fluoropyrimidine toxicity. Int. J. Cancer 136(3), 730-739 (2015).

50 Sistonen J, Büchel B, Froehlich TK et al. Predicting 5-fluorouracil toxicity: DPD genotype and 5,6-dihydrouracil:uracil ratio. Pharmacogenomics 15(13), 1653-1666 (2014).

- Study investigating relation between $D P Y D$ variants and dihydrouracil/uracil ratio.

51 Gamelin E, Boisdron-Celle M, Guérin-Meyer V et al. Correlation between uracil and dihydrouracil plasma ratio, fluorouracil (5-FU) pharmacokinetic parameters, and tolerance in patients with advanced colorectal cancer: a potential interest for predicting 5-FU toxicity and determining optimal 5-FU dosage. J. Clin. Oncol. 17(4), 1105 (1999). 
52 Jiang $\mathrm{H}$, Lu J, Jiang J, Hu P. Important role of the dihydrouracil/uracil ratio in marked interpatient variations of fluoropyrimidine pharmacokinetics and pharmacodynamics. J. Clin. Pharmacol. 44(11), 1260-1272 (2004).

53 Wettergren Y, Carlsson G, Odin E, Gustavsson B. Pretherapeutic uracil and dihydrouracil levels of colorectal cancer patients are associated with sex and toxic side effects during adjuvant 5-fluorouracil-based chemotherapy. Cancer 118(11), 2935-2943 (2012).

54 Kristensen MH, Pedersen P, Mejer J. The value of dihydrouracil/uracil plasma ratios in predicting 5-fluorouracil-related toxicity in colorectal cancer patients. J. Int. Med. Res. 38(4), 1313-1323 (2010).

55 Ben Fredj R, Gross E, Ben Ahmed S, Hassine H, Saguem S. The dihydrouracil/uracil ratio in plasma, clinical and genetic analysis for screening of dihydropyrimidine dehydrogenase deficiency in colorectal cancer patients treated with 5-fluorouracil. Pathol. Biol. 57(6), 470-476 (2009).

56 Largiadèr CR, Amstutz U, Froehlich TK et al. The dihydropyrimidine dehydrogenase gene as a major predictor of severe 5-fluorouracil toxicity: a classic reborn? Presented at: 2010 Meeting on Pharmacogenomics and Personalized Medicine. Cold Spring Harbor, NY, USA, 17-21 November 2010 .
57 Steimer W, Zöpf K, von Amelunxen S et al. Allele-specific change of concentration and functional gene dose for the prediction of steady-state serum concentrations of amitriptyline and nortriptyline in CYP2C19 and CYP2D6 extensive and intermediate metabolizers. Clin. Chem. 50(9), 1623-1633 (2004)

58 Gaedigk A, Simon SD, Pearce RE, Bradford LD, Kennedy MJ, Leeder JS. The CYP2D6 activity score: translating genotype information into a qualitative measure of phenotype. Clin. Pharmacol. Ther. 83(2), 234-242 (2008).

- The first study that allocated a quantitative score to a gene activity differentiation for CYP2D6.

59 Zhang X, Diasio RB. Regulation of human dihydropyrimidine dehydrogenase: implications in the pharmacogenetics of 5-FU-based chemotherapy. Pharmacogenomics 8(3), 257-265 (2007).

60 Mattison LK, Soong R, Diasio RB. Implications of dihydropyrimidine dehydrogenase on 5-fluorouracil pharmacogenetics and pharmacogenomics. Pharmacogenomics 3(4), 485-492 (2002).

61 Van Staveren MC, Guchelaar HJ, van Kuilenburg AB, Gelderblom H, Maring JG. Evaluation of predictive tests for screening for dihydropyrimidine dehydrogenase deficiency. Pharmacogenomics J. 13(5), 389-395 (2013). 